\title{
ARTICLES
}

\section{Study of the ultrahigh-energy primary-cosmic-ray composition with the MACRO experiment}

S. Ahlen, ${ }^{\mathrm{c}}$ M. Ambrosio, ${ }^{1}$ R. Antolini, ${ }^{\mathrm{b}}$ G. Auriemma, ${ }^{\mathrm{n}, *}$ A. Baldini, ${ }^{\mathrm{m}}$ B. B. Bam, G. C. Barbarino, ${ }^{1}$ B. C. Barish, ${ }^{d}$ G. Battistoni, ${ }^{f}$ R. Bellotti, ${ }^{a}$ C. Bemporad, ${ }^{m}$ P. Bernardini, ${ }^{j}$ H. Bilokon, ${ }^{f}$ V. Bisi, ${ }^{\mathrm{p}}$ C. Bloise, ${ }^{\mathrm{f}}$ S. Bussino, ${ }^{\mathrm{n}}$ F. Cafagna,${ }^{\mathrm{a}}$ M. Calicchio, ${ }^{\mathrm{a}}$ P. Campana, ${ }^{\mathrm{f}}$ D. Campana, ${ }^{1}$ M. Carboni, ${ }^{f}$ S. Cecchini, ${ }^{b,}{ }^{\dagger}$ F. Cei, ${ }^{\mathrm{m}}$ V. Chiarella, ${ }^{\mathrm{f}}$ C. Chiera, ${ }^{\mathrm{n}}$ A. Cobis, ${ }^{\mathrm{f}}$ R. Cormack, ${ }^{\mathrm{c}}$ A. Corona, ${ }^{\mathrm{n}}$ S. Coutu, ${ }^{\mathrm{d}}$ G. DeCataldo, ${ }^{\mathrm{a}}$ C. DeMarzo, ${ }^{\mathrm{a}}$ M. De Vincenzi, ${ }^{\mathrm{n}}$ A. Di Credico, ${ }^{\mathrm{i}}$ E. Diehl, ${ }^{\mathrm{k}}$ O. Erriquez, ${ }^{a}$ C. Favuzzi, ${ }^{a}$ D. Ficenec, ${ }^{c, \ddagger}$ C. Forti, ${ }^{f}$ L. Foti, ${ }^{n}$ P. Fusco, ${ }^{a}$ G. Giacomelli, ${ }^{b}$ G. Giannini, ${ }^{\mathrm{m}, \S}$ N. Giglietto, ${ }^{\mathrm{a}}$ P. Giubellino, ${ }^{\mathrm{p}}$ M. Grassi, ${ }^{\mathrm{m}}$ P. Green, ${ }^{\mathrm{r}}$ A. Grillo, ${ }^{\mathrm{f}}$ F. Guarino, ${ }^{1}$ C. Gustavino, ${ }^{g}$ A. Habig, ${ }^{\mathrm{h}}$ R. Heinz, ${ }^{\mathrm{h}}$ J. T. Hong, ${ }^{\text {d }}$ E. Iarocci, ${ }^{\mathrm{f}, * *}$ E. Katsavounidis, ${ }^{\text {d }}$ E. Kearns, ${ }^{\mathrm{c}}$ S. Klein, ${ }^{\text {c, }}{ }^{\dagger \dagger}$ S. Kyriazopoulou, ${ }^{\text {d }}$ E. Lamanna, ${ }^{\text {n }}$ C. Lane, ${ }^{\text {e C. Lee }},{ }^{k}$ D. Levin, ${ }^{k}$ P. Lipari, ${ }^{n}$ G. Liu, ${ }^{\mathrm{d}}$ R. Liu, ${ }^{\mathrm{d}}$ M. J. Longo, ${ }^{\mathrm{k}}$ G. Ludlam, ${ }^{\mathrm{c}}$ G. Mancarella, ${ }^{\mathrm{j}}$ G. Mandrioli, ${ }^{\mathrm{b}}$ A. Margiotta-Neri, ${ }^{\mathrm{b}}$ A. Marin, ${ }^{\text {c }}$ A. Marini, ${ }^{\text {f D. Martello, }}{ }^{\text {j }}$ G. Martellotti, ${ }^{\mathrm{n}}$ A. Marzari Chiesa, ${ }^{\mathrm{p}}$ M. Masera, ${ }^{\mathrm{p}}$ P. Matteuzzi, ${ }^{\mathrm{b}}$

D. G. Michael, ${ }^{\mathrm{d}}$ L. Miller, ${ }^{\mathrm{h}}$ P. Monacelli, ${ }^{\mathrm{i}}$ M. Monteno, ${ }^{\mathrm{p}}$ S. Mufson, ${ }^{\mathrm{h}}$ J. Musser, ${ }^{\mathrm{h}}$ S. Nutter, ${ }^{\mathrm{h}, \ddagger \ddagger}$ C. Okada, ${ }^{\mathrm{c}}$ G. Osteria, ${ }^{1}$ O. Palamara, ${ }^{\mathrm{j}}$ S. Parlati, ${ }^{\mathrm{g}}$ V. Patera, ${ }^{\mathrm{f}}$ L. Patrizii, ${ }^{\mathrm{b}}$ R. Pazzi, ${ }^{\mathrm{m}}$

C. W. Peck, ${ }^{d}$ J. Petrakis, ${ }^{q}$ S. Petrera, ${ }^{j}$ N. D. Pignatano, ${ }^{d}$ P. Pistilli, ${ }^{j}$ F. Predieri, ${ }^{b}$ L. Ramello, ${ }^{p}$ J. Reynoldson, ${ }^{\mathrm{g}}$ F. Ronga, ${ }^{\mathrm{f}}$ G. Rosa, ${ }^{\mathrm{n}}$ C. Satriano, ${ }^{\mathrm{n}, *}$ L. Satta, ${ }^{\mathrm{f} * *}$ E. Scapparone, ${ }^{\mathrm{b}}$ K. Scholberg, ${ }^{\mathrm{d}}$ A. Sciubba, ${ }^{n, * *}$ P. Serra Lugaresi, ${ }^{b}$ M. Severi, ${ }^{n}$ M. Sitta, ${ }^{p}$ P. Spinelli, ${ }^{a}$ M. Spinetti, ${ }^{f}$ M. Spurio, ${ }^{b}$ J. Steele, ${ }^{\mathrm{d}}$ R. Steinberg, ${ }^{\mathrm{e}}$ J. L. Stone, ${ }^{\mathrm{c}}$ L. R. Sulak, ${ }^{\mathrm{c}}$ A. Surdo, ${ }^{\mathrm{j}}$ G. Tarlé, ${ }^{\mathrm{k}}$ V. Togo,

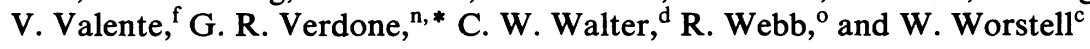

\section{(MACRO Collaboration)}

a Dipartimento di Fisica dell'Università di Bari and Istituto Nazionale di Fisica Nucleare, Bari, 70126, Italy

${ }^{\mathrm{b}}$ Dipartimento di Fisica dell'Università di Bologna and Istituto Nazionale di Fisica Nucleare, Bologna, 40126, Italy

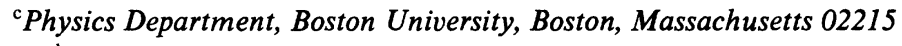

${ }^{\mathrm{d} C a l i f o r n i a}$ Institute of Technology, Pasadena, California 91125

'Department of Physics, Drexel University, Philadelphia, Pennsylvania 19104

${ }^{\mathrm{f}}$ Laboratori Nazionali di Frascati dell'Istituto Nazionale di Fisica Nucleare, Frascati, Roma, 00044, Italy

${ }^{\mathrm{g}}$ Laboratori Nazionali del Gran Sasso dell'Istituto Nazionale di Fisica Nucleare, Assergi, L'Aquila, 67010, Italy

${ }^{\mathrm{h}}$ Departments of Physics and Astronomy, Indiana University, Bloomington, Indiana 47405

'Dipartimento di Fisica dell'Università dell'Aquila and Istituto Nazionale di Fisica Nucleare, L'Aquila, 67100, Italy

'Dipartimento di Fisica dell'Università di Lecce and Istituto Nazionale di Fisica Nucleare, Lecce, 73100, Italy

${ }^{k}$ Department of Physics, University of Michigan, Ann Arbor, Michigan 48109

'Dipartimento di Fisica dell'Università di Napoli and Istituto Nazionale di Fisica Nucleare, Napoli, 80125, Italy

${ }^{\mathrm{m}}$ Dipartimento di Fisica dell'Università di Pisa and Istituto Nazionale di Fisica Nucleare, Pisa, 56010, Italy

${ }^{\mathrm{n}}$ Dipartimento di Fisica dell'Università di Roma and Istituto Nazionale di Fisica Nucleare, Roma, 00185, Italy

\footnotetext{
*Also Universitá della Basilicata, Potenza, 85100, Italy.

†Also Istituto TESRE/CNR, Bologna, Italy.

$\ddagger$ Now at Physics Department, Washington University, St. Louis, MO 63130.

§Also Universitá di Trieste and INFN, Trieste, 34100, Italy.

**Also Dipartimento di Energetica, Universitá di Roma,
}

Roma, 00185, Italy.

†'Now at Department of Physics, University of California, Santa Cruz, CA 95064.

$¥$ Currently at Laboratori Nazionali del Gran Sasso dell'INFN, 67010, Italy. 


\author{
${ }^{\circ}$ Physics Department, Texas A\&M University, College Station, Texas 77843 \\ ${ }^{\mathrm{P}}$ Dipartimento di Fisica dell'Università di Torino and Istituto Nazionale di Fisica Nucleare, \\ Torino, 10125, Italy \\ ${ }^{9}$ Bartol Research Institute, University of Delaware, Newark, Delaware 19716 \\ ${ }^{\mathrm{r}}$ Sandia National Laboratory, Albuquerque, New Mexico 87185
}

(Received 26 March 1992)

\begin{abstract}
We present the analysis of multiple-muon events collected with one supermodule (1013 $\mathrm{h}$ live time) and two supermodules (1195 h live time) of the MACRO detector at Gran Sasso, Italy. Multimuon rates are shown to be sensitive to primary-cosmic-ray energies between $\sim 50 \mathrm{TeV}$ and several thousand $\mathrm{TeV}$. Experimental data are compared with the expected rates from two composition models: a light (i.e., proton-rich) and a heavy (i.e., Fe-rich) composition. The predictions are based on a Monte Carlo simulation of the hadronic interactions of cosmic-ray nuclei, followed by a detailed tracking of the muons through the rock and the experimental apparatus. The results show good sensitivity of the MACRO detector to primary composition. The data exhibit a preference towards the light composition model.
\end{abstract}

PACS number(s): 96.40.Tv, 96.40.De, 98.70.Sa

\section{INTRODUCTION}

The elemental composition of the primary cosmic rays incident at the top of the Earth's atmosphere contains information about the nature of the cosmic-ray sources; the energy spectra of the different components are related to mechanisms of acceleration [1] and propagation in the interstellar medium [2]. The bulk of data about the elemental composition of cosmic rays comes from direct measurements with balloons and satellites. These experiments measure the absolute abundances of different nuclei and the energy spectra of the primary species up to energies of about $100 \mathrm{TeV}[3,4]$. Because of the steeply falling spectrum direct measurements at much higher energy require impractically large acceptance detectors and/or very long duration exposures. In the ultrahighenergy region $E_{0}>100 \mathrm{TeV}$, where the all-particle energy spectrum shows an abrupt steepening at about $3000 \mathrm{TeV}$ ("knee region"), very little is known about composition [5]. At present the only way to study this energy region is to obtain information indirectly from the analysis of the properties of secondary particles produced in atmospheric cascades. Indirect information on cosmic-ray primary composition can be extracted from surface measurements of the extensive air showers or from underground studies of the penetrating high-energy muon component. Both surface and underground detectors often have large acceptances and allow long exposure times.

The rates of muon bundles of different multiplicities measured in deep underground experiments are sensitive to the chemical composition and energy spectra of the primary cosmic rays, above a threshold determined by the rock overburden ( $\sim 50 \mathrm{TeV}$ at Gran Sasso). This sensitivity arises from the fact that heavy nuclei tend to generate a larger yield of charged pions and kaons, in the forward fragmentation (high-rapidity) region, than that of light nuclei [6], and it is these pions and kaons which can decay into the penetrating muons observed deep underground. At a fixed total energy, muons produced by light nuclei are more energetic than those from heavy parents, and hence they penetrate deeper into the earth. Above some threshold primary energy, however, the muons from heavy-nucleus interactions are sufficiently energetic to penetrate to the depth of the detector. The energy where this crossover occurs depends upon rock depth. Figure 1 shows the muon yield of proton and iron primaries at the average depth of MACRO, calculated from the parametrizations of Ref. [7].

With underground detectors, the energy and type of a primary cannot be determined on an event-by-event basis, but information on the mass composition can be obtained by comparing measured muon multiplicity distributions with those calculated with an accurate Monte Carlo simulation using trial models of the primary spectrum and composition. This procedure is complicated by our limited knowledge of the proton-nucleus and nucleusnucleus inclusive meson production and transverse momenta distributions at very high energies. While inclusive and total cross sections for $p-\bar{p}$ collisions have been measured up to $\sqrt{s} \sim 0.9 \mathrm{TeV}$ [8] and $1.8 \mathrm{TeV}$ [9], respectively, the data for nucleus-nucleus interactions ex-

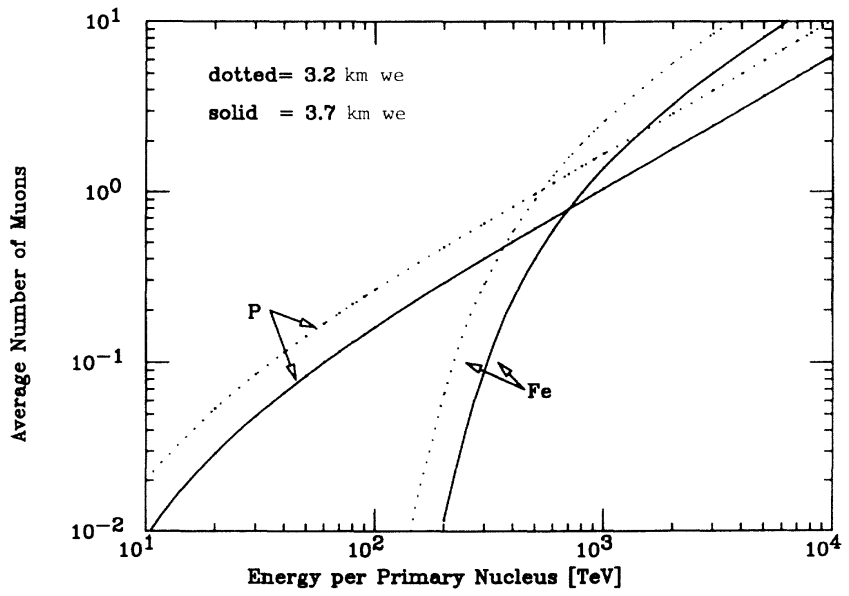

FIG. 1. Muon yield at two different depths for $p$ - or Feinduced showers as a function of total primary energy per nucleus, calculated from the parametrizations of Ref. [7]. Vertical incidence is assumed in the calculation. The solid line refers to the MACRO average depth, the dotted line to the minimum depth (see Sec. II). 
tend only up to a laboratory-frame energy of about 0.2 $\mathrm{TeV} /$ nucleon [10]. In the case of MACRO, where the minimum laboratory-frame primary energy required to efficiently produce observable muons is $\sim 50$ $\mathrm{TeV} /$ nucleus, properties of nuclear collisions must be extracted from the much lower-energy nucleus-nucleus data using phenomenological and theoretical considerations, with guidance from the $p-\bar{p}$ results. This process can introduce a degree of uncertainty in the interpretation of underground muon data (see Sec. IV B). The role of hadronic interactions is of great importance, particularly when the detector is not large enough to contain the entire muon bundle. The transverse momentum distribution of the energetic secondaries largely determines the fraction of muons above threshold that hit the detector. We have obtained excellent agreement between experimental data and a Monte Carlo simulation of the lateral distribution function of muons (the decoherence function) [11]. This success leads us to believe that we have an adequate understanding of the hadronic interactions.

Previous studies have been carried out with smaller detectors [12] but achieved limited sensitivity to different trial compositions. The MACRO effective detection area of $24 \mathrm{~m} \times 12 \mathrm{~m}$ is well suited for high statistics studies of muon bundles for which, at the Gran Sasso depth, the mean lateral separation is about $6 \mathrm{~m}$. About $10^{7}$ events will be collected per year with the full-sized detector (effective area $72 \mathrm{~m} \times 12 \mathrm{~m}$ ), thus allowing us to perform a study of multiple-muon physics at high muon multiplicities and large separations.

In this paper we present an analysis of multiple-muon events collected in MACRO with one supermodule (see Sec. II) operating from 4 March 1990 through 10 May 1990 and with two supermodules operating from 10 May 1990 through 19 July 1990 . The main purpose of this study is to investigate the sensitivity of MACRO to primary composition. To this end we compare our experimental data with a "light" and a "heavy" model (whose characteristics are specified in Sec. IV A). The same approach has also been used in other analyses [13].

In the next section we describe the experimental apparatus. In the third section we discuss the data selection criteria and the analysis method. The fourth section presents the details of the Monte Carlo simulation and the trial composition and hadronic interaction models used. In the last two sections we discuss the comparison between the measured distributions and the Monte Carlo predictions.

\section{THE EXPERIMENTAL SETUP}

The MACRO detector, located in hall B of the Gran Sasso National Laboratories in Italy, has a modular structure whose basic block is a "supermodule" of dimensions $12 \times 12 \times 4.8 \mathrm{~m}^{3}$ [14]. Each supermodule is equipped with streamer tube chambers, liquid scintillator tanks, and track-etch plastics. Individual supermodules are instrumented to operate independently, thus allowing completed sections of the detector to collect data, while other sections are under construction or undergoing tests.

The tracking system consists of ten horizontal layers of limited streamer tubes separated by $32 \mathrm{~cm}\left(\sim 60 \mathrm{~g} \mathrm{~cm}^{-2}\right)$ of rock absorber. Each tube is $12 \mathrm{~m}$ long and $3 \times 3 \mathrm{~cm}^{2}$ in cross section, and utilizes a $100 \mu \mathrm{m}$ anode wire and a graphite cathode. A two-dimensional readout is performed using the signals from the anode wires and external $26.5^{\circ}$ stereo angle strips. Each projective view has a spatial resolution of about $1.1 \mathrm{~cm}$. The intrinsic angular resolution is $0.2^{\circ}$ for muons crossing ten horizontal planes, considerably less than the mean multiple Coulomb scattering angle of muons in the rock $\left(0.6^{\circ}\right)$.

The minimum and average rock overburden above MACRO are 3200 and 3700 mwe (meters of water equivalent), respectively. The energy threshold at the Earth's surface, $E_{\text {th }}$ (in TeV), for a muon to cross a depth $h$ (in $\mathrm{km} w e$ ) [5] is determined by $E_{\mathrm{th}}$ $=0.53 \times\left(e^{0.4 \mathrm{~h}}-1\right)$. Above this energy, the survival probability is $30 \%$ or greater. $E_{\text {th }}$ is $\sim 1.4 \mathrm{TeV}$ for the minimum MACRO depth.

\section{DATA SELECTION AND EVENT ANALYSIS}

Muons are reconstructed separately in wire and strip views. A track is defined by alignment of at least four hits in different planes. For each event, we obtain two independent projected multiplicities $N_{W}$ and $N_{S}$, corresponding to the wire and strip views, respectively. In most cases, it was possible to associate tracks in the two views to reconstruct the muon path in three-dimensional space [11].

Our analyzed event samples correspond to 1013 and $1195 \mathrm{~h}$ of total live time for the sample with one supermodule (1SM sample) and two supermodules (2SM sample), respectively. For each event, we required that the average zenith angle of the muons be less than $60^{\circ}$. This conforms with the valid angular range of the muon generator parametrization, which assumes a geometrically flat Earth and atmosphere. The fraction of events surviving this cut was $\sim 94 \%$. When counting muons, we excluded nonparallel secondary particles produced by muon interactions in the surrounding rock or in the detector absorber. The parallelism cut selected muon pairs diverging by less than $3^{\circ}$. This is a very loose cut for the muon bundles at our depths, which have angular divergence around $1^{\circ}$ and are reconstructed with an accuracy at least three times better in most cases.

For a given event, the true multiplicity of muons transversing the detector can be different from $N_{W}$ and $N_{S}$ as reconstructed by the tracking algorithm, because of geometrical superposition of two or more tracks in one view, showering tracks, inefficiency or noise in the streamer tube system or inefficiency of the tracking algorithm. Events were visually scanned in order to resolve possible ambiguities. We assigned a range of different multiplicities to complicated events (mainly events with showers) when the true multiplicity could not be unambiguously determined. This was done by giving an equal fractional weight to each possible multiplicity within the range defined by the scanner. All events with $N_{W}>5$ or $N_{S}>5$ were scanned. This cutoff value is considerably lower than the multiplicity of about 10 , above which the tracking algorithm gives a less reliable multiplicity as- 
TABLE I. Muon multiplicities after scanning corrections. Corresponding live times are given in the

\begin{tabular}{cccccc}
\hline text. & \multicolumn{5}{c}{} \\
\hline \hline$N_{\mu}$ & $1 \mathrm{SM}$ & $2 \mathrm{SM}$ & $N_{\mu}$ & $1 \mathrm{SM}$ & 2 SM \\
\hline 1 & $123900 \pm 1300$ & $268700 \pm 6200$ & 13 & $0.6 \pm 0.6$ & $5.8 \pm 2.4$ \\
2 & $3920 \pm 130$ & $10400 \pm 250$ & 14 & $0.6 \pm 0.6$ & $4.3 \pm 2.1$ \\
3 & $658 \pm 45$ & $1620 \pm 69$ & 15 & $1.3 \pm 1.1$ & $1.3 \pm 1.1$ \\
4 & $148 \pm 14$ & $566 \pm 35$ & 16 & 0 & $1.7 \pm 1.3$ \\
5 & $78 \pm 10$ & $201 \pm 16$ & 17 & 0 & $2.2 \pm 1.5$ \\
6 & $38 \pm 9$ & $102 \pm 10$ & 18 & $1 \pm 1$ & $1.3 \pm 1.1$ \\
7 & $15 \pm 4$ & $69 \pm 9$ & 19 & & $0.5 \pm 0.5$ \\
8 & $15 \pm 4$ & $39 \pm 7$ & 20 & & $0.3 \pm 0.3$ \\
9 & $7 \pm 3$ & $19 \pm 5$ & 21 & & $0.3 \pm 0.3$ \\
10 & $5 \pm 2.2$ & $11 \pm 4$ & 22 & & $0.3 \pm 0.3$ \\
11 & $3.5 \pm 1.9$ & $8.5 \pm 2.9$ & & & 0 \\
12 & $1.3 \pm 1.1$ & $4.8 \pm 2.2$ & 27 & & $1 \pm 1$ \\
\hline \hline
\end{tabular}

signment. However, the chosen cutoff value resulted in not too large a sample to scan visually. The remaining events were scanned on a sampled basis. In this analysis a total number of about 5000 events were scanned. Based on the scanning results the number of events with multiplicity $N_{\mu}$ is given by

$$
n\left(N_{\mu}\right)=\sum f_{N_{W}, N_{S}}^{N_{\mu}} n\left(N_{W}, N_{S}\right)
$$

where $n\left(N_{W}, N_{S}\right)$ is the number of events reconstructed with multiplicities $N_{W}$ and $N_{S}$ and $f_{N_{W}, N_{S}}^{N_{\mu}}$ is the fraction of events in the $\left(N_{W}, N_{S}\right)$ subsample which were classified by visual scanning as having multiplicity $N_{\mu}$. The statistical uncertainty on $n\left(N_{\mu}\right)$ is therefore given by

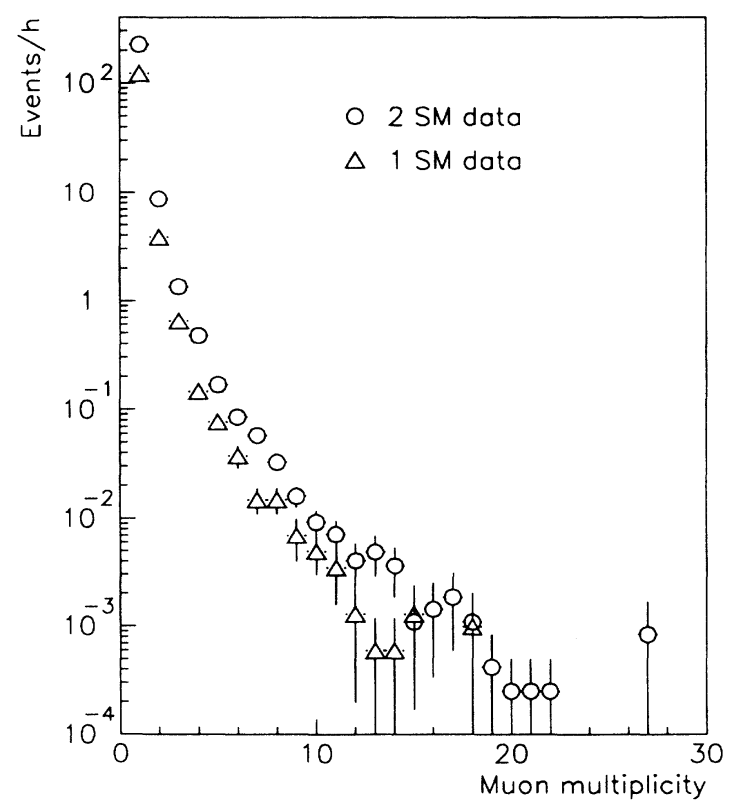

FIG. 2. Multimuon rates as a function of multiplicity for the one supermodule and two supermodule samples. Statistical errors, including scanning uncertainties, are shown.

$$
\begin{gathered}
\sigma^{2}=\sum\left[n\left(N_{W}, N_{S}\right)^{2} \frac{f_{N_{W}, N_{S}}^{N_{\mu}}\left(1-f_{N_{W}, N_{S}}^{N_{\mu}}\right)}{N_{N_{W}, N_{S}}}\right. \\
\left.+\left(f_{N_{W}, N_{S}}^{N_{\mu}}\right)^{2} n\left(N_{W}, N_{S}\right)\right],
\end{gathered}
$$

where $N_{N_{W}, N_{S}}$ is the number of scanned events for the $\left(N_{W}, N_{S}\right)$ subsample. The systematic error, evaluated with a partial double scanning, is of the same order as the statistical one.

The results obtained in the different periods of operation of the detector are reported in Table $I$. The difference in the fractional uncertainty on the number of single muons for the one and two supermodule data arises from the relatively small subsets of visually scanned events in these data samples. Statistical errors, inclusive of scanning uncertainties, are reported in this table. Figure 2 shows the multimuon rates for the one supermodule and two supermodule event samples. The increase of acceptance from one to two supermodules is reflected in an increase of muon rates and a sampling of higher multiplicities.

\section{MONTE CARLO SIMULATION}

Our Monte Carlo simulation has the following features.

(i) A physics generator including both a parametrization of hadronic interactions and the characteristics (energy spectrum and elemental composition) of the primary cosmic radiation.

(ii) A description of the rock depth distribution around the MACRO detector [15].

(iii) A GEANT [16] based simulation program describing the geometry and detector response of the experimental apparatus in detail and producing data with the same format as for real events. These data were processed using the standard off-line chain of analysis, thus allowing us to evaluate both the MACRO acceptance and the reconstruction program efficiency.

We generated primary-cosmic-ray nuclei with an ener- 
TABLE II. Normalization factor $K\left[\mathrm{~m}^{-2} \mathrm{~s}^{-1} \mathrm{sr}^{-1}(\mathrm{GeV} / \text { nucleus })^{\gamma-1}\right]$, slope $\gamma$, cutoff energy $E_{\text {cut }}$ $(\mathrm{GeV})$, and slope after cutoff $\gamma\left(E>E_{\text {cut }}\right)$ for the "light" and "heavy" models. The spectrum of each component is given by $d N / d E=K E^{-\gamma}$.

\begin{tabular}{ccccc}
\hline \hline Group & $K$ & $\gamma$ & $E_{\text {cut }}$ & $\gamma\left(E>E_{\text {cut }}\right)$ \\
\hline "Light" composition & & & & \\
$p$ & $1.50 \times 10^{4}$ & 2.71 & $2.0 \times 10^{4}$ & 3.0 \\
$\alpha$ & $1.87 \times 10^{3}$ & 2.50 & $3.0 \times 10^{6}$ & 3.0 \\
CNO & $5.69 \times 10^{3}$ & 2.71 & $3.0 \times 10^{6}$ & 3.0 \\
$\mathrm{Mg}$ & $3.30 \times 10^{3}$ & 2.71 & $3.0 \times 10^{6}$ & 3.0 \\
$\mathrm{Fe}$ & $2.60 \times 10^{3}$ & 2.71 & $3.0 \times 10^{6}$ & 3.0 \\
& $3.48 \times 10^{3}$ & 2.71 & $3.0 \times 10^{6}$ & \\
"Heavy" composition & & & & 3.0 \\
$p$ & & & & 3.0 \\
$\alpha$ & $1.50 \times 10^{4}$ & 2.71 & $1.0 \times 10^{5}$ & 3.0 \\
CNO & $5.69 \times 10^{3}$ & 2.71 & $2.0 \times 10^{5}$ & 3.0 \\
$\mathrm{Mg}$ & $3.30 \times 10^{3}$ & 2.71 & $7.0 \times 10^{5}$ & 3.0 \\
Fe & $2.60 \times 10^{3}$ & 2.71 & $1.2 \times 10^{6}$ & \\
\hline \hline
\end{tabular}

gy ranging from $3 \mathrm{TeV}$ up to $10^{5} \mathrm{TeV}$, which fully covers the energy region explored by MACRO (see Sec. IV A). The effect of the geomagnetic field, though visible in MACRO as a small asymmetry of at most $0.5 \mathrm{~m}$ in the muon pair separation distribution, produces negligible effects on multiplicity distributions because of the large detector size. For this reason, it has not been included in our Monte Carlo simulation.

\section{A. Primary spectra and composition models}

Direct measurements of the chemical composition of primary cosmic rays up to $100 \mathrm{TeV}$ have been recently presented by the Chicago group [3] and up to $900 \mathrm{TeV}$ by the JACEE Collaboration [4]. The measurements at the highest energies, derived by extensive air shower experiments, suffer from large uncertainties in the determination of the primary energy [5].

Several models for the primary composition have been proposed in order to explain the results of extensive air shower (EAS) arrays and emulsion chamber experiments. The various models differ both in the relative fraction of elements and in the shape of the all-particle spectrum, which determines the overall normalization of multimuon fluxes. In our analysis, we have chosen to use energydependent models constrained to reproduce the measured all-particle spectrum. Indeed, in order to impose selfconsistency to the trial models [17], it is important to constrain the compositions to reproduce the known abundances and spectra directly measured at $\leq 100 \mathrm{TeV}[3,4]$, and to agree with the extensive air shower measurements at higher energies. This normalization criterion can be somewhat debatable at the highest energies, again because the relationship between the measured shower size and the primary energy is not completely free from assumptions on the composition model.

In the present analysis we adopted this normalization procedure and used two trial compositions: the Maryland composition [18] ("heavy") and a low-energy composition (LEC) with an enhanced proton component [19] ("light") adjusted to give the same all-particle spectrum [17]. The characteristics of the two models are as follows.

"Light" model. An LEC model starting with a differential spectral index of 2.71 for all the species, with a flux adjusted addition of a pure protonic component above $20 \mathrm{TeV}$ and a fixed energy cutoff at $E_{\text {cut }}=3000$ $\mathrm{TeV}$.

"Heavy" model. An extrapolation of the Maryland model starting with a differential spectral index of 2.36 for iron and 2.71 for the other species and including a rigidity-dependent cutoff at $E_{\text {cut }}=100 Z \mathrm{TeV}$.

The basic parameters of the models are summarized in Table II. Figure 3 shows the fractional composition and Fig. 4 the all-particle spectrum for the two models superimposed on the available experimental data. In comparison, Fig. 5 shows the all-particle spectra for three widely used compositions, the constant mass composition (CMC) [24], and the Maryland [18] and Linsley [19] mod-
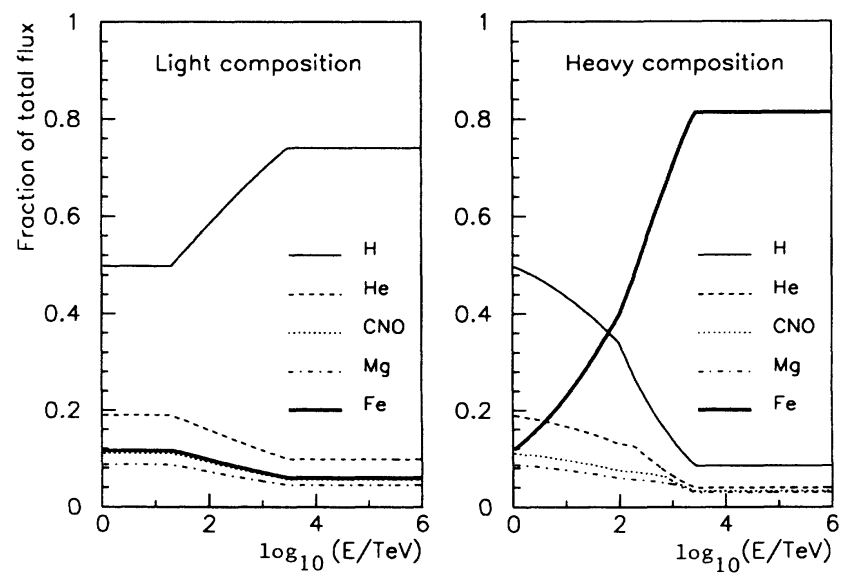

FIG. 3. Evolution of the fractional composition with energy for the "light" and "heavy" models [17], described in Sec. IV A. 


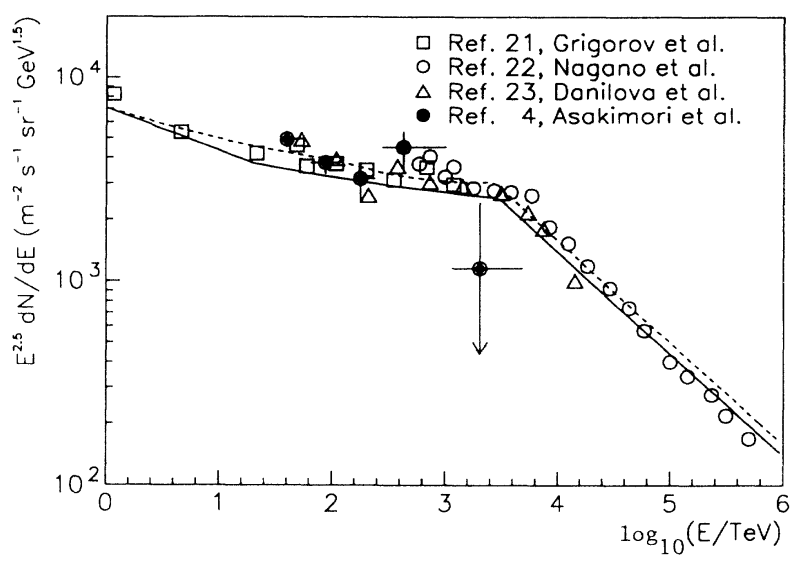

FIG. 4. All-particle energy spectrum for the "light" (solid line) and "heavy" (dashed line) models [17] superimposed on the available experimental data. Compilation of data taken from Hillas [20].

els. It can be easily seen that the normalization procedure we adopted [17] strongly reduces the differences in the energy spectra of the trial compositions.

It is important to know the window of primary energies explored by the detection of multiple muons. Figure 6 shows the calculated distributions of the primary energies that correspond to different detected multiplicities for each model. The mean primary energy for $N_{\mu} \geq 2$ is $\sim 1000 \mathrm{TeV}$ for the "light" and $\sim 1600 \mathrm{TeV}$ for the "heavy" model. The corresponding value for events with $\geq 6$ muons is $\sim 8000 \mathrm{TeV}$ for both compositions. Therefore, the majority of events in this analysis originate below the "knee," but the higher-multiplicity events are sensitive to composition above the "knee."

\section{B. Primary hadronic interaction models.}

The interpretation of deep underground muon data requires a simulation which includes the primary hadronic

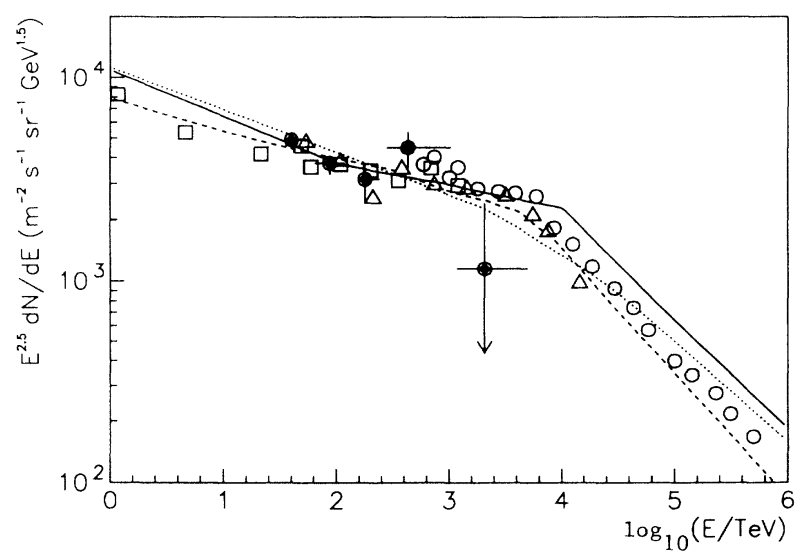

FIG. 5. All-particle energy spectrum for the Linsley [19] (solid line), Maryland [18] (dashed line), and CMC [24] (dotted line) models superimposed on the available experimental data (same as for Fig. 4).
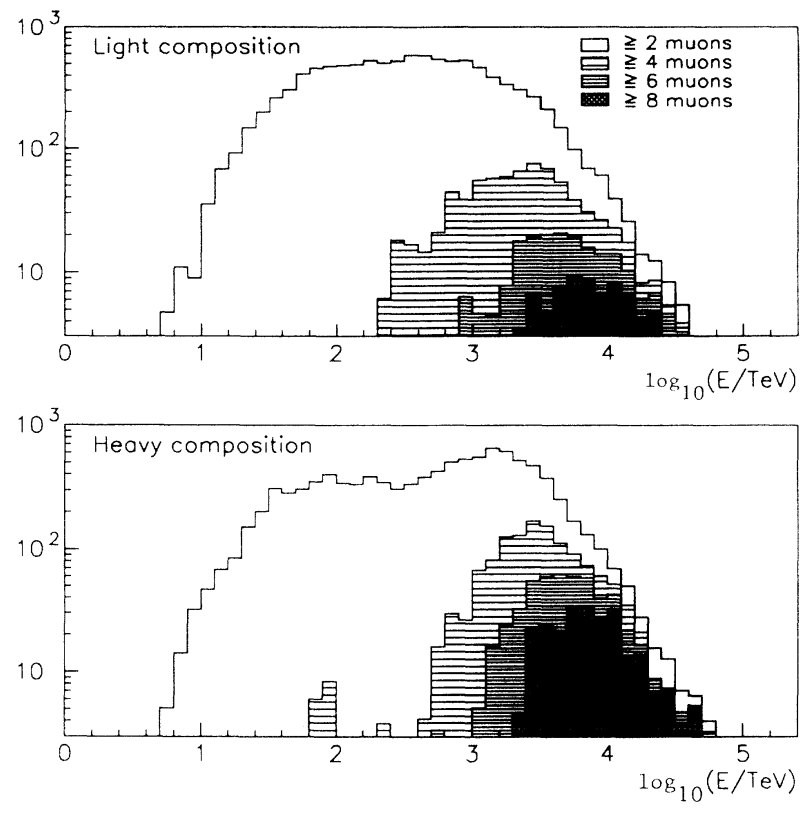

FIG. 6. Primary energies that correspond to different numbers of muons detected in MACRO for the "light" and "heavy" models.

interaction model, the air shower development, and the propagation of muons through the rock. The results of such a simulation are usually expressed in terms of parametrized formulas. These formulas describe the main features of the muon bundles as they would be observed in an infinite size detector, i.e., their lateral distance and multiplicity distributions as a function of the characteristics of the primary (mass, energy, and direction) and of the rock depth. These parametrizations can be used in Monte Carlo simulation programs in order to obtain predictions of multimuon data in the actual detector, at very little cost in CPU time.

A recent Monte Carlo simulation of atmospheric cascades [7] incorporates the most recent results from the CERN proton-antiproton collider (up to $\sqrt{s}=0.9 \mathrm{TeV}$ ) and the available measurements of nucleus-nucleus interactions from fixed target experiments. This interaction model describes multiple hadron production through a "multicluster" mechanism that reproduces the characteristics observed at the CERN collider energies and includes nuclear target effects. Extrapolation to higher energies is done in the context of inelastic " $\ln (s)$ physics." This simulation includes several differences from the previous standard parametrizations [6]. In particular, the probability of $N_{\mu}$ muons reaching the level of the detector deviates from a Poisson distribution and is better described by a negative binomial, which yields an increased probability of high-multiplicity events. Also the lateral distribution of the underground muons is different, the average muon separation being significantly larger in the model of Ref. [7]. Both models consider the nucleusnucleus interaction in the context of the "superposition model" [25]. A rough estimate of fragmentation effects on muon multiplicity distributions is given in Ref. [7]. 
More complete work on nuclear target effects is currently in progress [26].

A comparison of the measurements of the muon decoherence function of the MACRO multiple-muon events with Monte Carlo simulations [11] shows a better agreement with the more recent hadronic interaction parametrizations. In our simulation we therefore adopted this model [7].

\section{DISCUSSION}

The results of our full Monte Carlo simulations using the "light" and "heavy" composition models (described in Sec. IV A) are shown in Fig. 7 and compared with the experimental data for one and two supermodules.

Error bars on the data points of Fig. 7 represent the statistical uncertainty, including the uncertainty due to scanning. The uncertainties on the Monte Carlo predictions include several systematic effects.

(i) Uncertainties in the hadronic interaction model. No error estimate was provided for the parameters of the two aforementioned hadronic interaction models. The treatment of nuclear fragmentation has been suggested as one of the largest sources of uncertainties [7]. Compared to the superposition model, nuclear fragmentation yields deviations on multimuon rates which increase with multiplicity and are of the order of 10-20\% for $N_{\mu}=10$. To check the sensitivity of our Monte Carlo results to the hadronic interaction model, we compared the integral muon multiplicity distributions obtained using [7] and the older model of Ref. [6]. The differences ranged from $9 \%$ for a number of detected muons $N_{\mu} \geq 1$ to $30 \%$ for $N_{\mu} \geq 20$. We adopted these differences as an estimate of this systematic uncertainty.

(ii) Detector effects. We introduced into the detector simulation a streamer tube efficiency equal to $95 \%$ and a simulation of typical electronic noise in agreement with experimental observations. These instrumental effects produced negligible changes in the Monte Carlo predic-

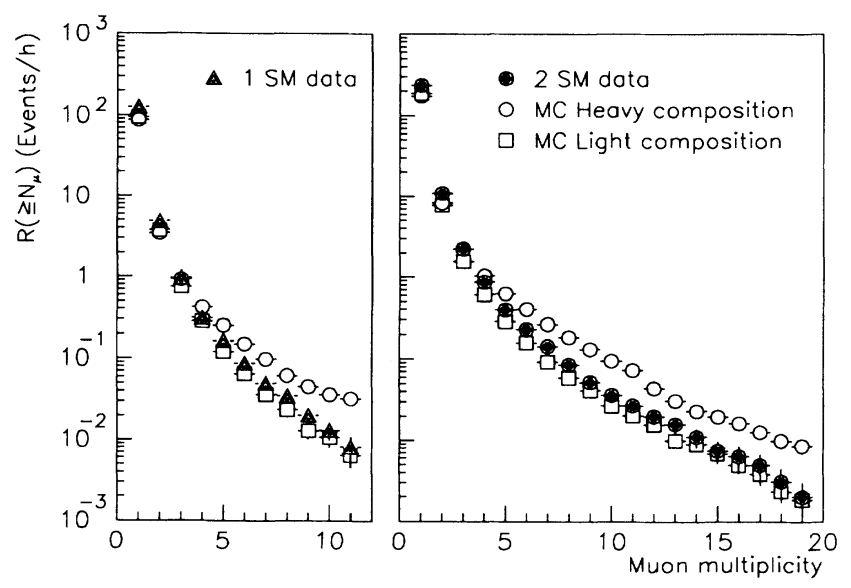

FIG. 7. Comparison between the integral multiplicity distributions of the one and two supermodule data and the Monte Carlo predictions. The Monte Carlo predictions for the "heavy" and "light" composition models include systematic uncertainties (see text). tions for muon multiplicity.

(iii) Uncertainty in the knowledge of the map of the rock around $M A C R O$. From a separate analysis of the flux of uncorrelated muons in MACRO [27], we estimate that the local uncertainty in the rock depth $h$ (in mwe) is of the order of a few percent. An uncertainty of $5 \%$ in $\Delta h / h$ was considered. The corresponding error on multimuon fluxes increased from $1 \%$ for $N_{\mu} \geq 1$ up to $10 \%$ for $N_{\mu} \geq 20$.

We also investigated the effect on the Monte Carlo predictions introduced by a net change of the rock depth by $1 \%$ everywhere. This change produced the same shift (from $6 \%$ to $25 \%$ in the rates of $N_{\mu} \geq 1$ and $N_{\mu} \geq 20$, respectively) on both curves of the Monte Carlo predictions, leaving our sensitivity to the two trial models unchanged.

The "light" and "heavy" models [17] used in this analysis are derived from the original Linsley [19] and Maryland [18] compositions, respectively, but are adjusted to the same all-particle spectrum as described in Sec. IV A. The muon multiplicity distributions obtained from the Maryland and Linsley compositions are very similar to those from the "heavy" and "light" models. Some deviations are observable for multiplicities exceeding about 10. This is due in part to the all-particle spectra of the original models, which differ from the ones used in this analysis mostly in the region above $\sim 10^{3} \mathrm{TeV}$.

The Baksan group [28] has recently reported a muon multiplicity analysis using a constant primary composition and constant spectral index (a CMC model), showing overall consistency with their data, as well as with the Frejus [29] and Nucleon Stability Experiment (NUSEX) [12] data. Their comparison is based on analytical calculations of the muon rates, at different rock depth, with a simplified description of the experimental apparatus. We have found that the multiplicity distribution calculated for MACRO with the CMC model [24] lies in between the "light" and "heavy" models, and is very close to the "light" composition in the low-multiplicity region $(\leq 6)$, where the data used in the Baksan analysis are statistically most significant. On the other hand, our present results are not very sensitive to whether there is an energy dependence to the composition. We will achieve better sensitivity when larger data samples of high-multiplicity events are analyzed.

\section{CONCLUDING REMARKS}

The general features of multiple-muon physics in MACRO are reproduced by Monte Carlo simulation. In particular, the simulations demonstrate MACRO's ability to discriminate between different cosmic-ray primary compositions normalized to render directly measured elemental abundances at $\leq 100 \mathrm{TeV}$. The observed muon multiplicity distribution, corresponding to a primary energy range of $50 \mathrm{TeV}$ up to several thousand $\mathrm{TeV}$, is more consistent with the light composition model.

A similar preference towards light composition has been recently reported [13]. The MACRO detector is being expanded to larger acceptance and will run for longer exposures. Future data samples with the full apparatus 
will allow us to continue this study and obtain more definite conclusions. A larger sample of high-multiplicity events will allow us to investigate correspondingly more energetic regions of the primary-cosmic-ray spectrum, above the "knee" region, where the knowledge about primary composition is still rather poor.

The combination of underground muon data with simultaneous surface measurements of the electromagnetic shower size also allows us to study the primary composition, and provides an estimate of the primary energy for each event [30].

\section{ACKNOWLEDGMENTS}

We are very grateful to T. K. Gaisser for helpful discussions and suggestions. We also gratefully acknowledge the continuous cooperation and support of the staff of the Gran Sasso National Laboratories and the technical support of our home institutions. The U.S. group acknowledges support from the U.S. Department of Energy and the National Science Foundation, and individual grants from NATO and the Istituto Nazionale di Fisica Nucleare.
[1] M. S. Longair, High-Energy Astrophysics (Cambridge University Press, Cambridge, England, 1981); R. Blandford and D. Eichler, Phys. Rep. 154, 1 (1987).

[2] C. J. Cesarsky, in Proceedings of the Twentieth International Cosmic Ray Conference, Moscow, USSR, 1987, edited by V. A. Kozyarivsky et al. (Nauka, Moscow, 1987), Vol. 8 , p. 87

[3] J. M. Grunsfeld et al., Astrophys. J. 327, L31 (1988); D. Müller et al., ibid. 374, 356 (1991).

[4] T. H. Burnett et al., Astrophys. J. 349, L25 (1990); K. Asakimori et al., in Proceedings of the 22nd International Cosmic Ray Conference, Dublin, Ireland, 1991, edited by M. Cawley et al. (Dublin Institute for Advanced Studies, Dublin, 1991), Vol. 2, p. 57.

[5] T. K. Gaisser, Cosmic Rays and Particle Physics (Cambridge University Press, Cambridge, England, 1990).

[6] T. K. Gaisser and T. Stanev, Nucl. Instrum. Methods A235, 183 (1985).

[7] C. Forti et al., Phys. Rev. D 42, 3668 (1990).

[8] G. J. Alner et al., Z. Phys. C. 32, 153 (1986).

[9] CDF Collaboration, in Proceedings of the Fourth International Conference on Elastic and Diffractive Scattering, La Biodola, Elba, Italy, 1991, edited by F. Cervelli and S. Zucchelli [Nucl. Phys. B (Proc. Suppl.) 25B, 19 (1992)].

[10] T. Akesson et al., Nucl. Phys. B333, 48 (1990).

[11] MACRO Collaboration, in Proceedings of the 22nd International Cosmic Ray Conference [4], Vol. 4, p. 567.

[12] G. Bologna et al., Nuovo Cimento 8C, 76 (1985); M. Aglietta et al., in Proceedings of the Topical Seminar on Astrophysics and Particle Physics, San Miniato, Italy, 1989, edited by F.-L. Navarria and P. G. Pelfer [Nucl. Phys. B (Proc. Suppl.) 14B, 193 (1990)].

[13] G. Auriemma et al., in Proceedings of the 22nd International Cosmic Ray Conference [4], Vol. 2, p. 101.

[14] M. Calicchio et al., Nucl. Instrum. Methods A264, 18 (1988); S. P. Ahlen et al., Phys. Lett. B 249, 149 (1990).
[15] The Gran Sasso rock map was derived by processing digitized data provided by the Istituto Geografico Militare.

[16] R. Brun et al., GEANT3 manual, CERN Report No. CERN DD/EE/84-1 (unpublished).

[17] G. Auriemma et al., in Proceedings of the 21st International Cosmic Ray Conference, Adelaide, Australia, 1989, edited by R. Protheroe (Graphic Services, Northfield, South Australia, 1990), Vol. 9, p. 362.

[18] J. A. Goodman et al., Phys. Rev. Lett. 42, 854 (1979); J. A. Goodman et al., Phys. Rev. D 26, 1043 (1982).

[19] C. Fichtel and J. Linsley, Astrophys. J. 300, 474 (1986); J. Linsley, in Proceedings of the 18th International Cosmic Ray Conference, Bangalore, India, 1983, edited by N. Durgaprasad et al. (TIFR, Bombay, 1983), Vol. 12, p. 135.

[20] A. M. Hillas, Annu. Rev. Astron. Astrophys 22, 425 (1984).

[21] N. L. Grigorov et al., Yad. Fiz. 11, 1058 (1970) [Sov. J. Nucl. Phys. 11, 588 (1970)].

[22] M. Nagano et al., J. Phys. G. 10, 1295 (1984).

[23] T. V. Danilova et al., in Proceedings of the 15th International Cosmic Ray Conference, Plovdiv, Bulgaria, 1977, edited by C. Ya. Christov et al. (Bulgarian Academy of Sciences, Sofia, 1977), Vol. 8, p. 129.

[24] J. Kempa and J. Wdowczyk, J. Phys. G 9, 1271 (1983).

[25] T. K. Gaisser et al., Phys. Rev. D 25, 2341 (1982).

[26] J. Engel et al., in Proceedings of the 22nd International Cosmic Ray Conference [4], Vol. 4, p. 1.

[27] MACRO Collaboration, in Proceedings of the 22nd International Cosmic Ray Conference [4], Vol. 4, p. 169.

[28] A. E. Chudakov et al., in Proceedings of the 22nd International Cosmic Ray Conference [4], Vol. 2, p. 5.

[29] Ch. Berger et al., Phys. Rev. D 40, 2163 (1989).

[30] MACRO and EAS-TOP Collaborations, in Proceedings of the 22nd International Cosmic Ray Conference [4], Vol. 4, p. 563, and Vol. 2, p. 61. 

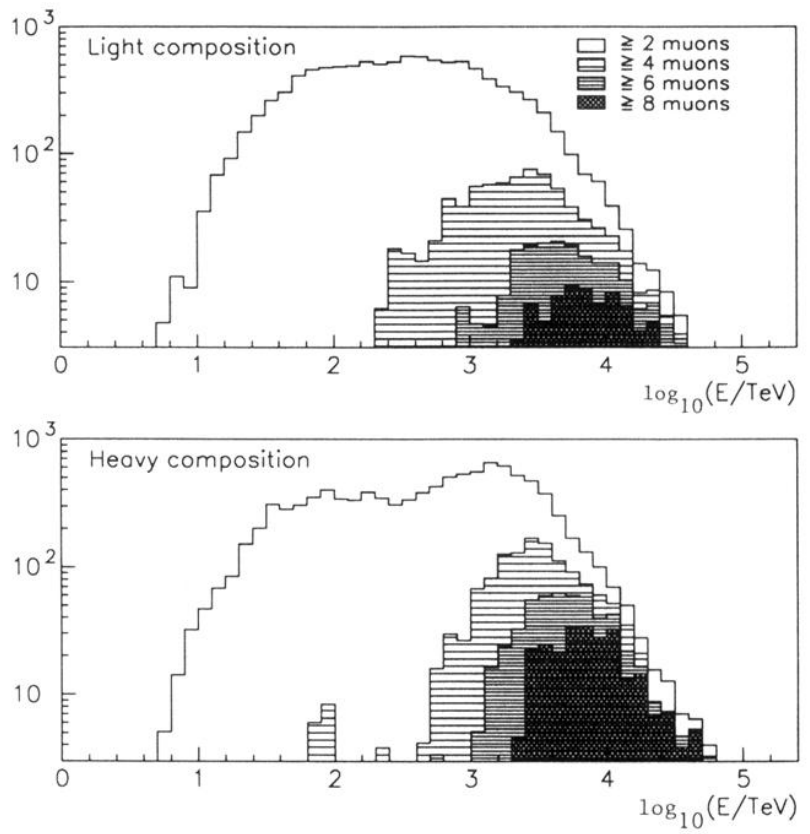

FIG. 6. Primary energies that correspond to different numbers of muons detected in MACRO for the "light" and "heavy" models. 